\title{
Friedrich A. HAYEK, The Market and Other Orders
}

\section{Michel Bourdeau}

\section{OpenEdition}

\section{Journals}

Édition électronique

URL : http://journals.openedition.org/ress/3150

DOI : $10.4000 /$ ress. 3150

ISBN : $1663-4446$

ISSN : 1663-4446

Éditeur

Librairie Droz

Édition imprimée

Date de publication : 15 novembre 2015

Pagination : 259-262

ISSN : 0048-8046

Référence électronique

Michel Bourdeau, "Friedrich A. HAYEK, The Market and Other Orders », Revue européenne des sciences sociales [En ligne], 53-2 | 2015, mis en ligne le 15 novembre 2015, consulté le 22 septembre 2020. URL : http://journals.openedition.org/ress/3150 ; DOI : https://doi.org/10.4000/ress.3150

Ce document a été généré automatiquement le 22 septembre 2020

(C) Librairie Droz 


\title{
Friedrich A. HAYEK, The Market and Other Orders
}

\author{
Michel Bourdeau
}

\section{RÉFÉRENCE}

Friedrich A. HAYEK, 2013, The Market and Other Orders, Bruce Caldwell (éd.), The Collected Works of F. A. Hayek, vol. XV, Chicago, The University of Chicago Press, $472 \mathrm{p}$.

1 Beaucoup d'ouvrages de Friedrich Hayek étant des recueils d'articles, les éditeurs des Collected Works (commencées en 1988 ; des quelque 20 volumes prévus, 15 ont déjà paru) ont pris le parti de redistribuer le tout de façon thématique. C'est ainsi que le présent volume rassemble, outre deux inédits publiés en appendices, 16 articles, qui couvrent une période de près de 40 ans (1937-1975) et sont regroupés, par ordre chronologique, en 3 parties.

2 Précédée d'un court essai de 1965, Kinds of Rationalism, qui sert d'introduction à l'ensemble, la première partie, The Early Ideas (1937-1948 ; 4 chapitres, qui figuraient dans Individualism and Economics,1948), met en place la plupart des thèmes développés par la suite, et contient certains des textes les plus célèbres de Hayek, comme Économie et Connaissance (1937) ou Sur la compétition (1948), qui en est comme le corollaire. Les Faits des sciences sociales (1943) s'en prend au naturalisme scientiste et développe le point de vue subjectif caractéristique de l'école autrichienne: pour l'économiste, le sucre se définit non par ses propriétés chimiques, mais par le fait que les gens croient qu'il possède un pouvoir édulcorant.

3 La deuxième partie, From Chicago to Freiburg, further Developpment (1955-1963; 4 chapitres qui figuraient dans Studies in Philosophy, Politics and Economics, 1967, dont la traduction française n'est ni faite ni à faire) marque un changement d'orientation. Un seul texte porte sur l'économie (et encore: dans ses rapports à la politique), la leçon inaugurale de Fribourg, en 1963 ; pour le reste, on y trouve The Rule of Law, une série de 
conférences prononcées au Caire quand l'Egypte était encore un royaume, et deux essais de philosophie des sciences, où perce l'intérêt de l'auteur pour la psychologie.

La troisième et dernière partie, A General Theory of Order, with Applications (1964-1975 ; 8 chapitres, repris en 1978 dans New Studies in Philosophy, Politics, Economics and History of Ideas), est la plus longue mais aussi la plus diverse. La dimension épistémologique passe au premier plan et, aux thèmes déjà signalés, viennent s'ajouter des réflexions sur la théorie de l'évolution (1967), sur la primauté de l'abstrait (1969) ou sur la complexité (1964). L'appendice A (50 p.) A New Look at Economic Theory, reproduit une série de 4 conférences données à l'université de Virginie en 1961 et restées jusqu'alors inédites. Une présentation de l'éditeur, B. Caldwell, d'une quarantaine de pages, complète l'ensemble.

5 Le principe des œuvres complètes veut que s'y côtoient le meilleur et, sinon le pire, du moins le moins bon; de même, il est difficile d'y éviter les redites. Mais le plus frappant, c'est la diversité des sujets abordés: ainsi, dans Rules, Perception and Intelligibility (chap. 8, 1962), Hayek invoque tour à tour la linguistique, l'éthologie, la psychologie de la forme, l'esthétique et la biologie. Le fil directeur, donné par le titre, n'en est pas moins solide. Le passage du phénomène économique du marché à des considérations beaucoup plus générales nous met en face de ce qui fait l'originalité de Hayek. Ce dernier occupe en effet une position singulière; à commencer chez les économistes: à Chicago, en 1945, ceux-ci n'avaient pas voulu recruter l'auteur de $L a$ Route de la servitude et il lui fallut se contenter d'une chaire de social research, financée par des fonds privés. Inversement, il portait sur la plupart de ses collègues un jugement sévère : celui qui n'est qu'économiste ne peut pas être un bon économiste (p. 229). La grandeur de Hayek et l'influence prodigieuse que sa pensée a exercé dans le dernier demi siècle tiennent à ce qu'il a été plus qu'un économiste et qu'il a vu, dans l'ordre spontané créé par la main invisible, le fondement et des sciences sociales et du libéralisme. Laissant de côté ses incursions dans l'épistémologie, il suffira ici de s'en tenir à ce qui touche à l'idée d'ordre spontané et à ses corollaires immédiats (concurrence et subjectivisme), ainsi qu'aux idées d'évolution et de règle, qui lui sont liées.

6 Article séminal, Économie et Connaissance (chap. 1) marque, aux dires de l'intéressé, le moment où il est entré véritablement en possession de sa pensée : ce qui s'échange sur le marché, ce ne sont pas seulement ni même d'abord des marchandises, mais plus fondamentalement des informations. Le système des prix fixés par le marché utilise la totalité des connaissances dispersées, sert la totalité des différentes fins individuelles et fournit ainsi l'exemple d'une coordination sans centralisation, d'un ordre spontané, qui surgit sans avoir été voulu. D'où le rapport avec les jeux : le résultat est pour une part aléatoire (p. 310); et aussi avec l'abstrait: le résultat n'est pas intentionnel, il est produit par dès règles abstraites, qui s'appliquent de façon aveugle, et ne favorisent personne en particulier. Pour bien comprendre ce qu'est l'ordre spontané du marché, encore appelé "catallaxie", il convient de ne pas le confondre avec ce que Hayek appelle une "économie" et qui relève d'un autre type d'ordre, celui des " organisations ». Une organisation poursuit un but commun et se donne pour cela une échelle de fins hiérarchisées. Dans une catallaxie en revanche, il n'y a pas de but commun et chacun poursuit son intérêt particulier. Si l'on peut parler de "merveille " (p. 101), c'est que le résultat est pourtant optimum et que, de plus, tout le monde est traité de la même façon. 
7 Écrit trente ans plus tard, La Concurrence comme méthode de découverte (chap. 12) développe des idées convergentes. La concurrence, c'est le moteur du marché, le mécanisme par lequel passe la transmission de la connaissance et qui permet de coordonner sans centraliser. Certes, il y a concurrence sans marché et les concours, les compétitions, montrent que celle-ci est bien une procédure de découverte; mais le marché repose sur la mise en concurrence des marchands. Les mêmes idées sont développées, en négatif, dans Les Erreurs du constructivisme, (chap. 14, 1970), un des essais favoris de l'auteur, pour qui dénoncer les erreurs du sens commun constituait une des principales tâches de l'économiste. En l'occurrence, il s'agit ici de faire valoir, contre ceux à qui il reproche de singer les sciences de la nature, le point de vue subjectif caractéristique de l'école autrichienne.

8 Le marché n'est toutefois qu'un exemple d'ordre spontané parmi d'autres et la notion demande à être étudiée pour elle-même. Deux concepts nouveaux apparaissent alors : ceux de règle et d'évolution, ainsi qu'un problème récurrent, celui de la genèse des ordre spontanés dans les sociétés. Entre ordre spontané et évolution, le rapport est posé comme immédiat : les deux idées sont jumelles (p. 289 ; l'idée est donnée comme empruntée à Carl Menger, qui posait l'élément génétique comme constitutif des sciences théorétiques); mais ce qu'on peut dire alors est très général : l'économie est plus proche de la biologie que de la physique, et en physique, de la cosmologie que de la mécanique. Pour en savoir davantage, il faut passer par le concept de règle. On connait l'importance accordée par les libéraux à l'idée de règle du droit (Rule of Law ; voir ici chap. 6, p. 119-194); il ne s'agit toutefois que d'une forme raffinée (au sens ou le sucre est raffiné), d'un phénomène plus général qu'il importe de saisir dans toute sa portée. Si l'ordre spontané est un produit de l'action humaine, et non d'un dessein humain ou d'une action visant ce résultat, comment en comprendre l'apparition? La réponse passe par l'idée de règle de conduite: l'action humaine est guidée par des règles. L'ordre spontané résulte de l'action humaine qui à son tour résulte de règles de conduite, étant entendu que l'ordre résultant n'est pas plus voulu par les règles qu'il n'est voulu par les agents. Il importe alors de voir que si, quand on explique un ordre spontané, on suppose données les règles qui le produisent, celles-ci n'ont rien d'inné, elles sont le produit d'une évolution, et c'est sur l'évolution des règles beaucoup plus que sur celle des ordres spontanés qu'il convient de s'interroger (chap. 10, Notes on the Evolution of Systems of Rule of Conduct). Sur ce point, Hayek se réclame de Darwin, tout en soutenant que le darwinisme a précédé Darwin (note p.301) et se trouve déjà en substance dans les Lumières écossaises. L'évolution procède par sélection, mais la sélection des règles est indirecte, via le succès des ordres spontanés qui en résultent (p. 282-284). D'où cette thèse très remarquée: si la transmission des règles se fait d'individu à individu (génétiquement ou culturellement), la sélection est une sélection de groupe. Ce faisant, Hayek prenait parti dans un débat qui divise les biologistes : ce qui est sélectionné par la sélection naturelle, sont-ce des individus ou des groupes?

Si Hayek se conçoit comme un héritier de la révolution marginaliste, il se reconnaît beaucoup moins volontiers en revanche dans la mathématisation de l'économie qui l'a souvent accompagnée et qui a emprunté à la physique la forme d'une théorie de l'équilibre. À cette dernière, il reproche de reposer sur des hypothèses problématiques (p. 384) et, plus encore, sur une conception de la concurrence qui la viderait de tout contenu. Du coup, on ne peut plus parler d'équilibre, mais seulement d'un optimum : une situation où les chances pour un individu choisi au hasard d'atteindre son but sont 
maximales (p. 307-310). On peut toutefois se demander en quel sens il s'agirait d'un optimum et il n'est pas nécessaire d'être constructiviste au sens de Hayek pour voir qu'un ordre spontané est susceptible d'être modifié et amélioré. La difficulté est à rattacher à la confusion grossière opérée entre le couple naturel-artificiel et le couple naturel-conventionnel (ou positif: phusei/thesei; voir par exemple p. 293-295), qui induit une approche biaisée de notre pouvoir d'intervention sur l'ordre spontané entendu dans toute sa généralité. Pour le dire autrement, ce qui manque à Hayek est une théorie satisfaisante de la Technè, comme le montrent les pages qu'il consacre aux rapports de l'économie et de la technologie (p. 402-414).

Quoi qu'on pense des idées défendues dans ce livre, elles ont du moins l'immense mérite de nous aider à comprendre le monde dans lequel nous vivons aujourd'hui, et qu'elles ont puissamment contribué à façonner. Si, en 1948, Hayek pouvait écrire (p. 115) : " the current tendency in discussion is to be intolerant about the imperfections [of competition] and to be silent about the prevention of competition ", il semble bien que ce soit aujourd'hui tout le contraire: on a choisi de fermer les yeux sur toutes ses imperfections et la toute puissante autorité de la concurrence est là pour ramener dans le droit chemin ceux qui songeraient à s'en écarter.

\section{AUTEURS}

\section{MICHEL BOURDEAU}

Université Paris-1 - IHPST / CNRS / ENS 